\title{
PREVALENCE OF ANXIETY WITHIN THE FIRST YEAR AFTER STROKE A SYSTEMATIC REVIEW AND META-ANALYSIS
}

Anna Danielsson, Lena Rafsten, Katharina Stibrant Sunnerhagen

Dep Clinical Neuroscience, Rehabilitation Medicine

anna.danielsson@neuro.gu.se

\section{TAKE HOME MESSAGE}

A PREVALENCE OF ANXIETY

AFTER STROKE OF $30 \%$ WAS FOUND, WHICH IS HIGHER THAN PREVIOUSLY SHOWN

INTERVENTIONS TO PREVENT OR TREAT ANXIETY SHOULD BE CONSIDERED

\section{Background}

Anxiety is associated with decreased quality of life and depression, but gets less attention than other psychological consequences after stroke. Updated knowledge regarding prevalence of anxiety is warranted in order to identify a possible need for specific rehabilitation interventions.

\section{Aim}

To estimate the prevalence of anxiety in the first year after stroke.

\section{Methods}

Literature searches in EMBASE, MEDLINE, PsycINFO, Cochrane Library, Amed and CINAHL were conducted in May 2015 and April 2017.

Inclusion criteria:

- Consecutive sample

- Hemorrhagic/ ischemic stroke/ transient ischemic attack

- Anxiety categorized on a rating scale

- First year after stroke

Exclusion criteria:

- Intervention study

- Selected population

- Report by proxy

Two reviewers screened and included studies and assessed quality using a checklist. Meta-analyses using the random effects model were conducted.

Heterogeneity was estimated using the Q-test and $\mathrm{I}^{2}$.

\section{Results}

Of 4453 titles screened, 37 studies comprising a total of 13756 participants fulfilled criteria. Study populations had mean ages from 52 to 79 years. Anxiety was assessed between 2 days and 1 year after stroke. The Hospital Anxiety and Depression Scale- Anxiety (HADSA) was used in 31 studies while the rest used other scales. The cut-offs for HADS-A ranged from $>6-\geq 11$. Most studies were of medium quality.

The overall pooled prevalence of anxiety at any time during the first year was $29.3 \%$ [95\% Cl $25.1 \%-33$. $\left.5 \%),\left(I^{2}=97 \%, p<0.00001\right)\right]$. Pooling the 31 studies using HADS-A showed a prevalence of $27.4 \%[95 \% \mathrm{Cl}$ $\left.22.6-32.1 \%\left(I^{2}=97 \%, p<0.00001\right)\right]$ (Fig. 1$)$.

The prevalence 0-2 weeks post stroke was $34.8 \%$ [95 $\%$ Cl $24.9-44.6 \%$ ], 2 weeks - 3 months $23.2 \%$ (95\% Cl 16.3-30.1\%) and $3-12$ months $35.6 \%$ [ $95 \%$ Cl $17.7-$ 54.3] (Fig. 2).

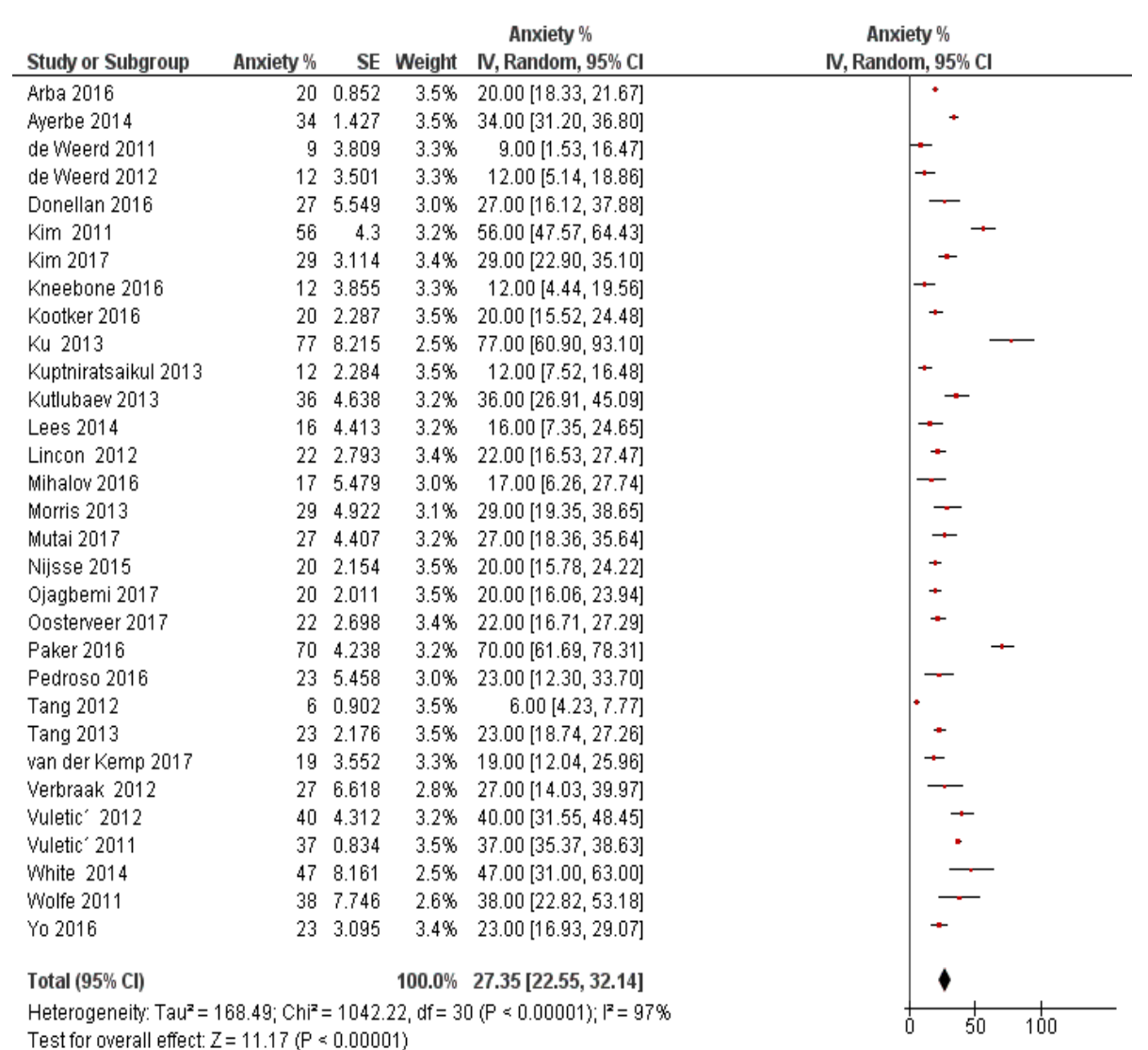

Figure 1. Prevalence of anxiety within the first year

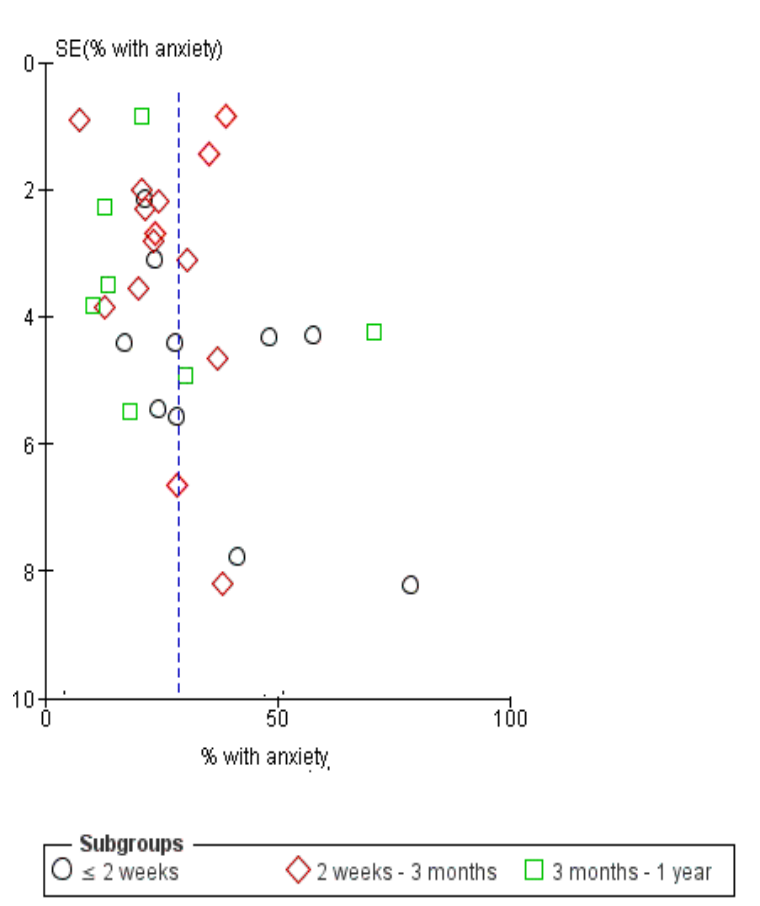

Figure 2. Funnel plot of studies based on time period 